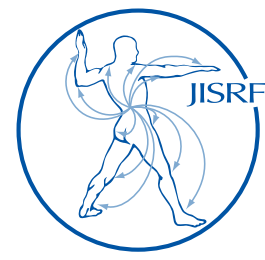

\title{
Pendulum Test: A Highly Accurate and Simple Physical Examination Maneuver to Identify Hip Pathology
}

Oshima $Y^{1}$, Fetto $J^{1}$

\begin{abstract}
Background: Patients with hip pathology often complain of various symptoms, e.g. pain or discomfort of low back, lower extremity, hip, groin, thigh, buttock or knee. Physicians may be distracted by these complaints, and misdiagnose and mistreat hip pathology. To avoid this, the pendulum test, which is performed with a patient seated on the examination table and hips and knees are flexed at 90 degrees, while the examiner passively swings the patient's lower extremity in and out as a pendulum, has been employed for all patients with the complaint of low back, hip and knee.

Objective: The efficacy and the accuracy of the pendulum test were evaluated.

Patients and methods: Consecutive 40 patients, who had complained pain or discomfort of low back, lower extremity, hip, groin, thigh, buttock and knee were examined by the pendulum test.

Results: Eighteen patients were positive for the pendulum test, and all of them correlated to the hip pathology.

Conclusions: The pendulum test was confirmed to be easily performed and reliable in detecting the hip pathology. Therefore, this test is highly recommended for the differentiation of patients with low back, hip and knee complaints.
\end{abstract}

Keywords: pendulum test; hip joint; internal and external rotation; physical examination Level of Evidence: AAOS Therapeutic Level IV

\section{Introduction}

The physical examination of hip joints includes evaluation of the range of motion with some classical procedures, e.g. Patrick's test and Thomas test. Positive results of the test are often followed by the diagnostic imaging examinations, i.e. the roentgenogram, CT scanning and MR imaging. Patients with hip pathology, however, do not always complain of hip pain. The pain or discomfort of low back, lower extremity, groin, thigh, buttock or knee are all com-
1 Division of Adult Reconstructive Surgery, Department of Orthopaedic Surgery, New York University Hospital for Joint Diseases, 1040 First Avenue, \#345, New York, NY 10022 USA (Direct reprint requests to Yasushi Oshima)

\section{(C) 2015 Oshima, Fetto. All rights reserved}

Authors retain copyright and grant the journal right of first publication with the work. Reconstructive Review follows the Creative Commons Attribution-NonCommercial CC BY-NC. This license allows anyone to download works, build upon the material, and share them with others for non-commercial purposes as long as they credit the senior author, Reconstructive Review, and the Joint Implant Surgery \& Research Foundation (JISRF). An example credit would be: "Courtesy of (senior author's name), Reconstructive Review, JISRF, Chagrin Falls, Ohio". 
mon complaints, which may be caused by hip pathology. Moreover, the hip joint is deep-seated and difficult to be palpated compared to the spine and the knee. For these reasons, the physicians sometimes misdiagnose and treat the wrong part of the body, instead of hip joint.

To avoid this inaccuracy, we have been employed the pendulum test, for all patients with complaints of low back, hip and knee pain or discomfort as an accurate maneuver to identify the presence of the hip pathology. This test has been used as one of the maneuver to diagnose and manage patients prior to hip surgery [1]. We have found that this test is highly efficient for the diagnosis of even early stage hip pathology. The objective of this study was to document the efficacy of the pendulum test to detect the hip pathology for the patients with low back, hip and knee pain.

\section{Maneuver of Pendulum Test}

Once a patient is suspected of pathology involving lumbar spine, hip or knee joints, after taking the detailed clinical history, we perform the pendulum test at the beginning of the physical examination. The patient is sitting on the edge of the examination table, bilateral hips and knees are flexed at 90 degrees and the feet are off the ground. The examiner sits in front of the patient, holds the knee gen- tly with one hand, and grabs the ipsilateral ankle with the other hand. Then, the examiner gently swings the patient's lower extremity in and out as a pendulum (Fig. 1). This in and out motion demonstrates external and internal rotation of the hip joint, respectively. Usually this test is performed on the asymptomatic side as a base line, before examining the contralateral limb.

The test is regarded as positive, when a patient demonstrates discomfort, pain, or a noticeable difference in the range of motion in the hip joints. If it is accompanied by pain or discomfort on the ipsilateral hip or groin, it indicates the existence of hip joint pathology. If the hip rotation is limited, especially the internal rotation, this is the evidence of hip anatomically advanced pathology.

\section{Patients and Methods}

Consecutive 40 patients in our outpatient clinic, who had complained pain or discomfort of low back, lower extremity, hip, groin, thigh, buttock, and knee were examined.

Patients were 25 to 103 years old, average was $68 \pm$ 15 years old, and were 14 males and 26 females. Of 40 patients, the chief complaints were; 21 with knee pain or discomfort, 8 with groin pain, 3 with hip pain, 1 with low
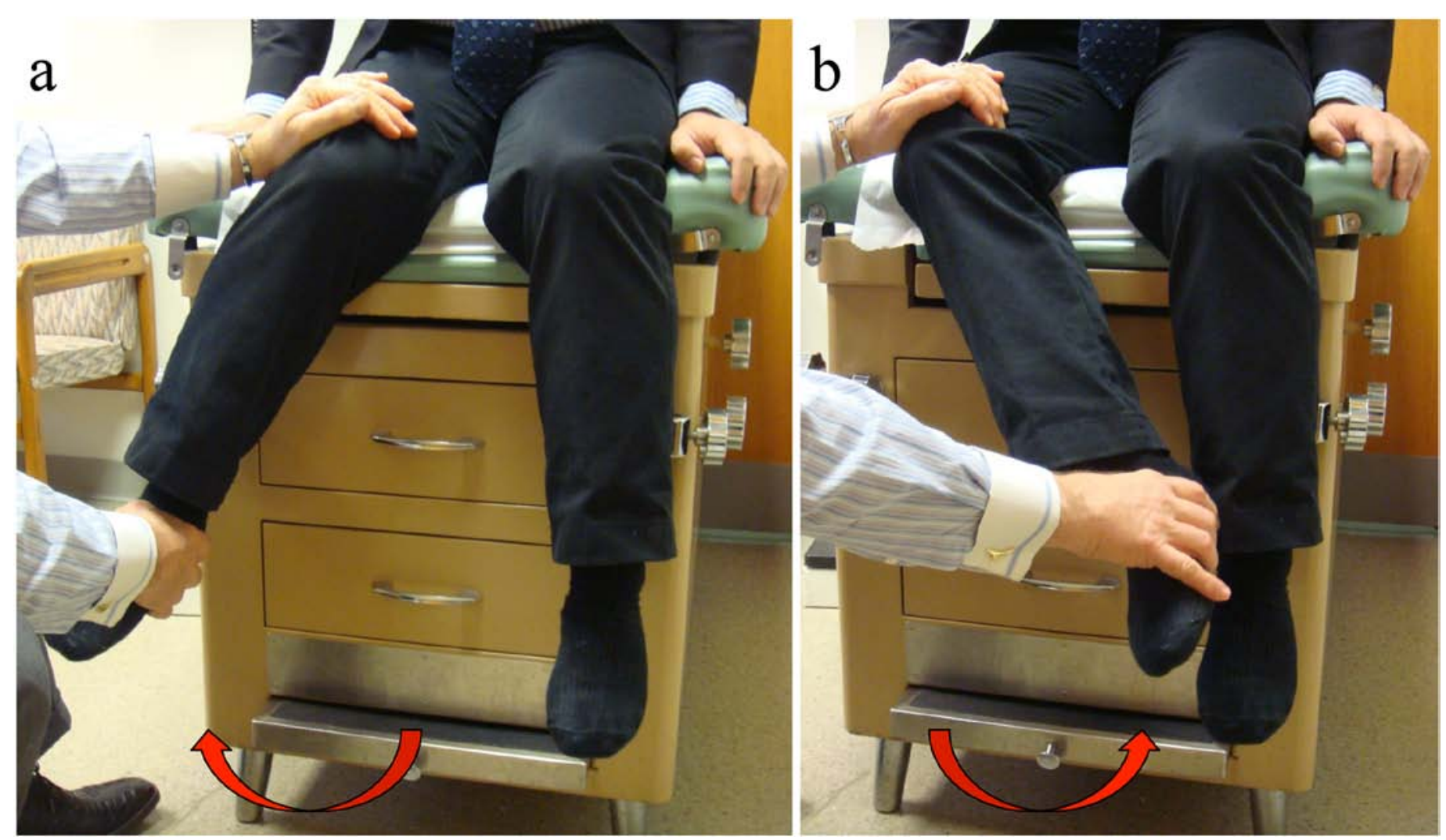

Fig. 1 : Maneuver of the pendulum test. The internal rotation (a) and external rotation $(b)$ of hip joint under the flexion at 90 degrees of the hip and knee is demonstrated. 
back pain, 1 with lower back and lower extremity pain, 2 with low back and hip pain or discomfort, 1 with low back and groin discomfort, 1 with groin, thigh and buttock discomfort, 1 with buttock and lower extremity pain, and 1 with lower extremity pain.

This study was approved by Institution Review Board (IRB). No funding or financial benefits provided to the authors for the project from any source.

\section{Results}

Twenty two patients were negative for the pendulum test and 18 patients were positive. There were 21 patients who presented with a complaint of knee pain or discomfort. Of these, 2 had a positive pendulum test. They were found to have aseptic loosening post total hip arthroplasty and osteoarthritis (OA) of the hip. All 8 patients with groin pain and 3 patients with hip pain had a positive pendulum test. All were shown to have diagnosed with hip related diseases. Eight patients had a complaint of low back pain, together with the pain of hip, groin, thigh, buttock or lower extremity. Three had a negative pendulum test and $5 \mathrm{had}$ a positive test. Especially for these cases, the pendulum test was extremely an effective tool for differentiating hip from low back pathologies in spite of their complex presentation. Although some cases had both OA of the spine and the hip, it was possible to distinguish which was the more critical complaint in these patients. Consequently, all patients with the positive pendulum test correlated to the hip pathology.

\section{Case reports}

\section{CASE 1}

A patient was a 66 years old male, and the chief complaint was intermittent discomfort of bilateral knees while walking, greater in the right than left. He had suffered an insidious increase in symptoms for one year, without any history of prior trauma. Because of the tenderness of the medial side of both knees, he was diagnosed as internal derangement of the knees, however, the knee joints had a full of range of motion without swelling or effusion. Moreover, there was no objective finding consistent with OA of the knee evaluated by the roentgenogram and MR imaging examinations. The conservative treatment of the knees had not been effective, and thus he was referred to our clinic for a second opinion evaluation. Although his knees were examined in great detail, the referring physician had performed no examination for the hips.
He presented independently ambulating without a cane, however, his right lower extremity was slightly externally rotated while walking. The pendulum test was performed, and he complained of discomfort in both hip joints, greater in the right than left, especially when the right with internal rotation. The arc of movement was decreased bilaterally more in the right compared with the left. Therefore, hip pathology was suspected. The roentgenogram findings of the hip joints showed the early OA with a slight osteophyte formation and a joint space narrowing (Fig. 2), confirming the efficacy of pendulum test.

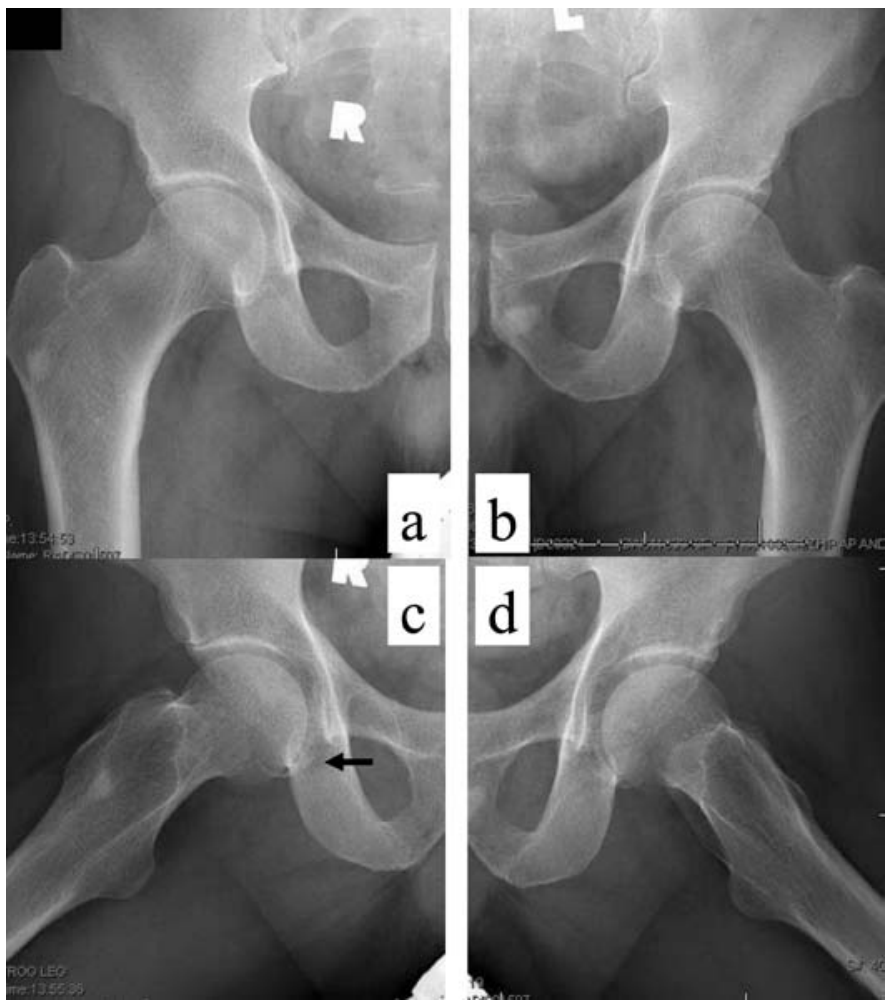

Fig. 2 : The roentgenogram findings of anterior-posterior view $(a, b)$ and lateral view $(c, d)$ of bilateral hip joints. Osteophyte formation and joint space narrowing were slightly detected in the right hip at the lateral view (arrow).

\section{CASE 2}

A patient was a 55 years old male with a chief complaint of left groin pain. He had developed increasing intermittent discomfort in the left groin, aggravated with activities and restricting range of motion over many years. He was status post spinal fusion of the fifth lumbar and first sacral vartebras. The primary doctor suspected the cause of the groin discomfort was due to increasing demand on hip range of motion following the spinal fusion. However, the hip joints had not been evaluated. The patient presented to our institution for a second opinion evaluation.

With the pendulum test, the patient complained of the discomfort with internal rotation of the hip, greater in the 
left than right. Further the test showed limitation of internal rotation of both hip joints. He was asymptomatic on the right, and the limitation was more severe on the left, however, the internal rotation was also limited on the right. The roentgenogram evaluation showed that mild OA on the left hip and minimal OA in the right hip, besides the post status of spinal fusion surgery (Fig.3,4), again confirming the diagnostic accuracy of the pendulum test.

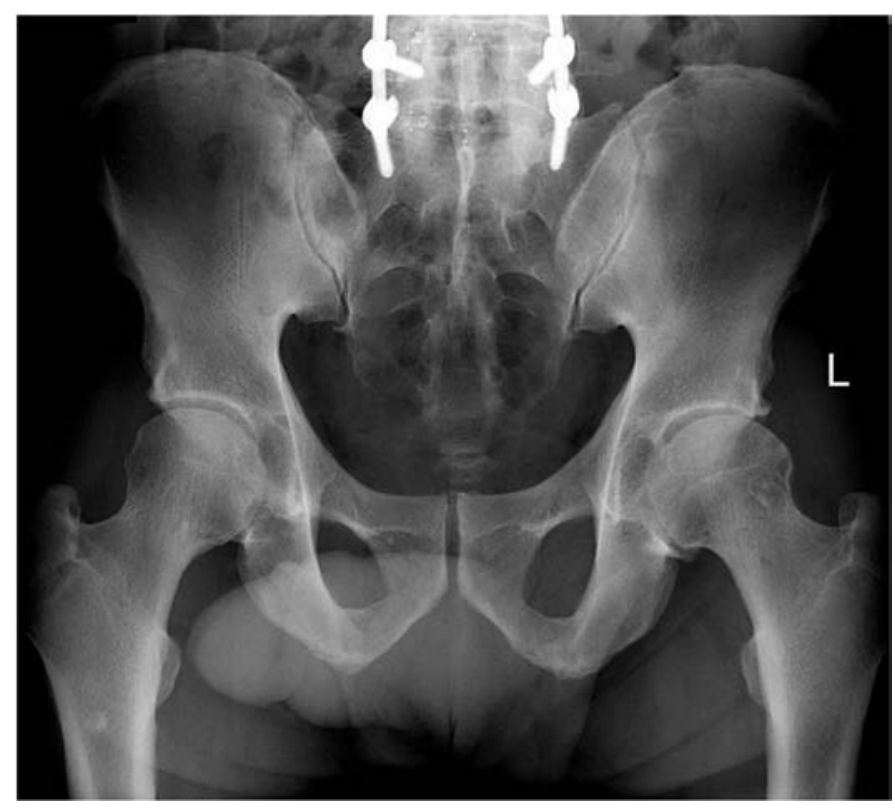

Fig. 3 : The roentgenogram findings of pelvis, post spinal fusion surgery.

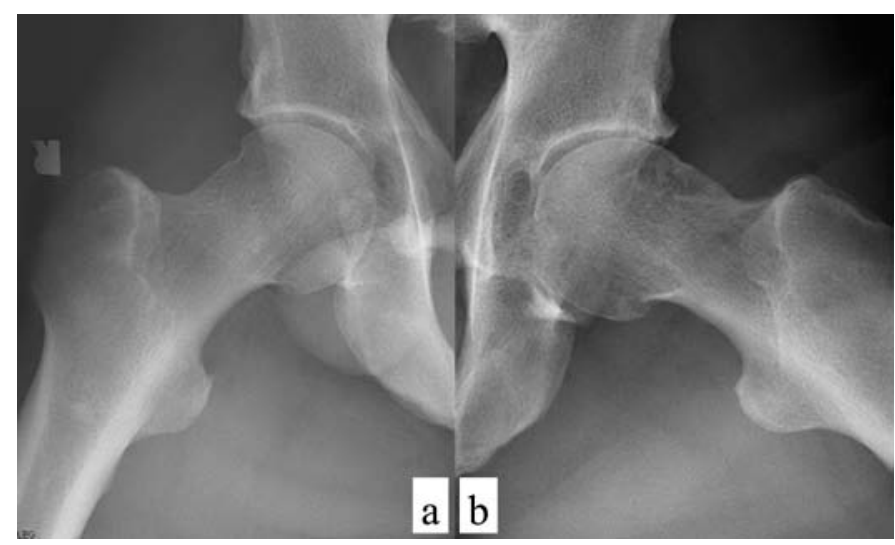

Fig. 4 : The roentgenogram findings of lateral view $(a, b)$ of bilateral hip joints. Mild OA on the left hip and minimal OA in the right hip were detected.

\section{Discussion}

In outpatient clinics, physicians are often misled by the location of the patients' complaints of pain or discomfort. Although the pathology emanates from the hip joint, patients sometimes complain of the low back, thigh or knee joint pain. For this reason, physicians may focus on the complaint sites, and unfortunately have their attention drawn away from the true site of pathology, leading to misdiagnosis and mistreatment. Conversely, even if a physician suspects hip pathology, and begins with an examination of the hip joint, patients who complain of low back or knee joint may feel uncomfortable and distrustful, and may lose confidence in the physician believing their complaints come from the symptomatic sites of which they are complaining.

The lumbar spine, hip and knee joints are anatomically related and work synergetically during activity of daily living. Because of its simplicity, accuracy, and efficacy, we routinely perform the pendulum test, with the patient seated, at the beginning of physical examination for patients with low back, hip and knee complaints. This test is easily performed without the patients' awareness that the hip joints are being assessed. Thus, we could evaluate hip joints accurately with the patients' in a relaxed condition. Once the pendulum test is positive, the meaning of the test is explained as an evaluation of hip joints, and further physical and imaging examinations of hip joints are continued.

The hip as a "ball-and-socket" joint provides no geometric resistance to the rotational torque. Thus, rotational movement is totally dependent upon a static ligamentous structure (especially the iliofemoral ligament) and the dynamic action of the gluteus medius. The iliofemoral ligament, also is known as the Y-shaped ligament, is the largest ligament in the body and tightens with internal rotation $[2,3]$. When the hip joint is inflamed, synovial liquid increases, the capsular ligaments become swollen, and the intra-articular pressure rises. These reactions cause tightening of the capsule and are manifested as acute groin discomfort. To reduce the symptom, patients will reflexively assume an external rotational posture to relax the capsular ligaments [2]. As a consequence of their external rotational positioning of the lower extremity, the ipsilateral knee is subjected to increase torsional stress, as seen in the first case. The knee is not designed to tolerate this torsion force because the knee is a "hinge" joint. This transfer of rotation force from the damaged hip to the otherwise normal knee causes pain and stress at both the medial and the patellofemoral components of the knee. We diagnosed this first case as OA of the hip joint, with pain at the knee due to secondary mechanical stress. In the second case, both the patient and the physician believed the groin pain had caused by the restricted lumbar motion following the spinal fusion surgery. However, the pendulum test was positive, proving the groin pain was due to OA of the hip.

There have been many tests described for the evaluation of the hip. The Patrick's test, also known as the Faber test, 
is often performed to evaluate with the hip and the sacroiliac joint. The tested hip is simultaneously flexed, abducted, externally rotated, and extended with the subject supine by the examiner. While holding the contralateral anterior superior iliac spine, the ipsilateral knee is stressed to lowered towards the table. If the knee does not lower to the table, it is suspected as a hip flexion contracture or protective illiopsoas spasm. If the ipsilateral hip pain is reproduced, it is suggestive of the hip joint disorder. If the pain is elicited on the contralateral side, it is suggestive of the sacroiliac joint disorder. The Thomas test is used to demonstrate a hip flexion contracture. The patient brings one knee to the chest with subject supine. If the patient's contralateral hip flexes, it indicates the tightness of iliopsoas, tensor fascia latae, or rectus femoris. Femoroacetabular impingement (FAI) is a condition of subtle developmental abnormalities of the hip $[4,5]$. The flexion-internal rotation-adduction impingement test to detect FAI is performed by the examiner with the subject supine. If the pain is reproduced, it is suggestive of FAI. The accuracy of these tests has been proven [6-9].

These examinations of the hip joint require the patient to lie supine and prone on the examination table. However, it is difficult to assume these postures for the patients with low back, hip or knee symptoms, flexion contracture of the hip joints, or after some surgical procedures. Therefore, a test for hip pathology with a patient seated, offers much advantage. OA of the hip starts with the deterioration of the internal rotation $[10,11]$. The most frequent location for FAI is the anterior rim area and the most critical motion is the internal rotation of the hip at 90 degrees flexion [12]. Thus, the internal rotation is the important morton to detect the early stage of OA and FAI, which can be accurately demonstrated by the pendulum test. It is also maybe performed in patients after total hip replacement and femoral head replacement without the risk of dislocation.

A limitation of this study is a small number of patients evaluated. However this test has been performed on many patients in our institution. We have been satisfied with the ease with which it can elicit the hip discomfort due to a diseased hip joint, even at a very early stage of OA. This is the first article to discuss the examination maneuver based on the anatomy and pathology of the hip joints. The pendulum test was considered to be a very accurate and useful maneuver in the evaluation of hip joints, even when patients have a simultaneous pathology at low back, hip or knee.

\section{Conclusions}

We have found that limited rotation, particularly internal rotation, of the hip joint while a patient is seated with the hip and knee flexed at 90 degrees is extremely predictive of hip pathology. The positive pendulum test highly correlated to the hip pathology.

\section{References}

1. Stone M, Assessment of the difficult primary hip replacement, Curr Orthop. 2008 Jun 22;3:165-172

2. Gray H, Articulations of the lower extremity, Gray's Anatomy, Fifteenth edition, 1995, 240-246.

3. Martin HD, Savage A, Braly BA, Palmer IJ, Beall DP, Kelly B. The function of the hip capsular ligaments: a quantitative report. Arthroscopy. 2008;24(2):188-195.

4. Myers SR, Eijer H, Ganz R. Anterior femoroacetabular impingement after periacetabular osteotomy. Clin Orthop Relat Res. 1999;363:93-99.

5. Ganz R, Parvizi J, Beck M, Leunig M, Notzli H, Siebenrock KA. Femoroacetabular impingement: a cause for osteoarthritis of the hip. Clin Orthop Relat Res. 2003;417:112-120.

6. Theiler R, Stucki G, Schütz R, Hofer H, Seifert B, Tyndall A, Michel BA. Parametric and non-parametric measures in the assessment of knee and hip osteoarthritis: interobserver reliability and correlation with radiology. Osteoarthritis Cartilage. 1996;4(1):35-42.

7. Cibere J, Thorne A, Bellamy N, Greidanus N, Chalmers A, Mahomed N, Shojania K, Kopec J, Esdaile JM. Reliability of the hip examination in osteoarthritis: effect of standardization. Arthritis Rheum. 2008;15;59(3):373-381.

8. Martin RL, Sekiya JK., The interrater reliability of 4 clinical tests used to assess individuals with musculoskeletal hip pain. J Orthop Sports Phys Ther. 2008;38(2):71-77.

9. Lee KM, Chung CY, Kwon DG, Han HS, Choi IH, Park MS. Reliability of physical examination in the measurement of hip flexion contracture and correlation with gait parameters in cerebral palsy. J Bone Joint Surg Am. 2011;19;93(2):150-158.

10. Croft PR, Nahit ES, Macfarlane GJ, Silman AJ. Interobserver reliability in measuring flexion, internal rotation, and external rotation of the hip using a plurimeter. Ann Rheum Dis. 1996;55(5): 320-323.

11. Holla JF, van der Leeden M, Roorda LD, Bierma-Zeinstra SM, Damen J, Dekker J, Steultjens MP. Diagnostic accuracy of range of motion measurements in early symptomatic hip and/or knee osteoarthritis. Arthritis Care Res. 2012 Jan;64(1):5965 .

12. Ganz R, Leunig M, Leunig-Ganz K, Harris WH. The etiology of osteoarthritis of the hip: an integrated mechanical concept. Clin Orthop Relat Res. 2008;466(2):264-272. 